\title{
Percutaneous biportal endoscopic decompression for lumbar spinal stenosis: a technical note and preliminary clinical results
}

\author{
Jin Hwa Eum, MD, ${ }^{1}$ Dong Hwa Heo, MD, PhD, ${ }^{1}$ Sang Kyu Son, MD, ${ }^{2}$ and \\ Choon Keun Park, MD, PhD'
}

'Department of Neurosurgery, Spine Center, The Leon Wiltse Memorial Hospital, Suwon; and 2Department of Neurosurgery, Spine Center, Gumi Kang-dong Hospital, Gumi, Korea

\begin{abstract}
OBJECTIVE The use of conventional uniportal spinal endoscopic decompression surgery for lumbar spinal stenosis can be limited by technical difficulties and a restricted field of vision. The purpose of this study is to describe the technique for percutaneous biportal endoscopic decompression (PBED) for lumbar spinal stenosis and analysis of clinical postoperative results.
\end{abstract}

METHODS The authors performed a unilateral laminotomy with bilateral foraminal decompression using a unilateral biportal endoscopic system in patients with single-level lumbar stenosis. The authors enrolled only patients who underwent follow-up for longer than 12 months after PBED. Fifty-eight patients were enrolled in this study. This approach was based on 2 portals: one portal was used for continuous irrigation and endoscopic viewing and the other portal was used to manipulate the instruments used in the decompression procedures. Clinical parameters such as the Oswestry Disability Index (ODI), Macnab criteria, and postoperative complications were analyzed.

RESULTS Neural decompression was effectively performed in all enrolled patients. The mean ODI was significantly lower after PBED. Of 58 patients, $47(81.0 \%)$ had a good or excellent result according to the Macnab criteria. Postoperative ODI and visual analog scale scores were significantly improved compared with preoperative values.

CONCLUSIONS From a surgical point of view, percutaneous biportal endoscopy is very similar to microscopic spinal surgery, permitting good visualization of the contralateral sublaminar and medial foraminal areas. The authors suggest that the PBED, which is a minimally invasive procedure, is an alternative treatment option for degenerative lumbar stenosis.

http://thejns.org/doi/abs/10.3171/2015.7.SPINE15304

KEY WORDS biportal endoscopy; lumbar stenosis; endoscopic decompression

$\mathrm{T}$ Traditionally, lumbar stenosis is treated with an open decompressive laminectomy, a foraminotomy, or fusion. ${ }^{2,6,8,9}$ Recently, minimally invasive spinal surgical methods have developed to improve preservation of the surrounding normal anatomical structures, such as the muscles and ligaments..$^{5,9,11}$ Microscopic bilateral decompression via a unilateral approach has been used in the treatment of lumbar spinal stenosis., ${ }^{9,11}$ Percutaneous endoscopic interlaminar decompression for lumbar stenosis remains a challenging procedure even for an experienced endoscopic surgeon.,12 Additionally, vision is restricted and technical difficulties can arise in spite of using a mi- croscope or uniportal spinal endoscope. Our technique of percutaneous biportal endoscopic decompression (PBED) is a modification of percutaneous uniportal interlaminar epidural endoscopic surgery. The PBED method is based on the same operative technique as other surgical procedures, such as ipsilateral microscopic laminotomy and bilateral decompression, with patients in the prone position. Compared with open microscopic spinal surgery, the PBED technique can reduce muscle injury and allows excellent visualization of the contralateral traversing root. In this article, we introduce and describe the technique for PBED in the treatment of lumbar spinal stenosis. 


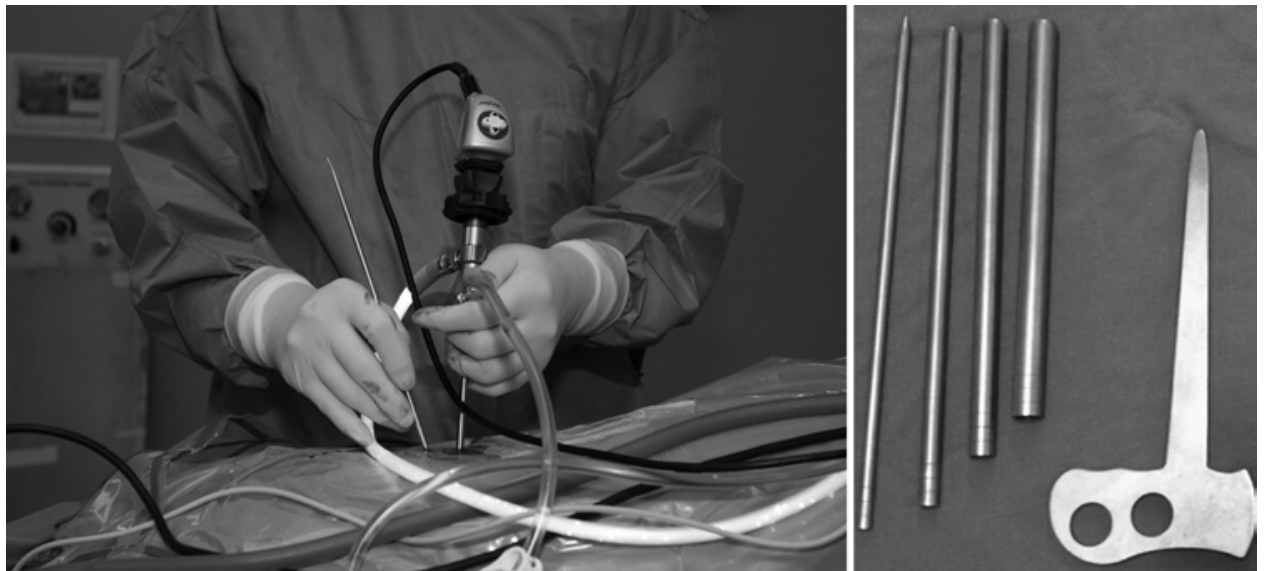

FIG. 1. Equipment used in unilateral biportal endoscopic decompression. A $0^{\circ} 4$-mm-diameter arthroscope (left). Specially designed dissector for exposure of the interlaminar space and serial dilators (right).

\section{Methods}

We performed PBED at 12 levels (from L2-3 to L5S1) on 3 cadavers before clinical application. We have performed more than 50 cases of endoscope-assisted laminotomy and discectomy for lumbar disc herniation. After accumulating experience with the biportal endoscopic procedures, we started PBED for lumbar spinal stenosis.

\section{Equipment Used in the Percutaneous Biportal Endoscopic Procedure}

During the procedures, we used a $3.5-\mathrm{mm}$ spherical bur (Conmed Linvatec), $0^{\circ}$ 4-mm-diameter arthroscope (Conmed Linvatec), bipolar flexible radiofrequency probe (Ellman Trigger-Flex probe, Ellman International), 3.5mm VAPR radiofrequency electrode (DePuy Mitec), serial dilators, a specially designed dissector, a pressure pump irrigation system (Smith \& Nephew, Inc.), and standard laminectomy instruments, such as hook dissectors, Kerrison punches, and pituitary forceps (Fig. 1).

\section{Surgical Procedure}

The PBED procedure is similar to a knee arthroscopic or thoracoscopic surgery. Two portals were used: one portal was used for continuous irrigation and endoscopic viewing and the other portal was used for insertion and manipulation of the instruments used in decompression procedures (e.g., laminotomy and complete removal of the ligamentum flavum) (Figs. 1 left and 2). The procedures were performed under general or epidural anesthesia with the patient in a prone position, with a Jackson table or radiolucent Wilson frame used to minimize abdominal pressure. A waterproof surgical drape was applied after induction of anesthesia.

The target pathological stenotic level was identified under fluoroscopic guidance. The exact target point was the intersection between the lower lamina margin and 1 $\mathrm{cm}$ lateral to the ipsilateral spinous process, as determined through the associated lateralizing symptoms. In the absence of lateralizing signs or symptoms, a left-sided approach is preferred for a right-handed surgeon. A $1.0-\mathrm{cm}$ skin incision (caudal portal) was made vertically above the target point (Fig. 2). A K-wire was introduced through the skin incision in the direction of the target point. Serial dilators were inserted toward the lower lamina. Following removal of the dilators, a specially designed dissector was moved to the lower lamina. Interlaminar soft tissue was dissected laterally to the medial margin of the facet capsule. A second 1-cm incision for the endoscope (cranial portal) was made by transverse insertion of a cannula $6 \mathrm{~mm}$ in diameter, approximately $2-3 \mathrm{~cm}$ above the upper edge of the first caudal skin incision (Fig. 2). A $0^{\circ}$ endoscope was inserted through the cranial portal after insertion of the cannula. A saline irrigation pump was

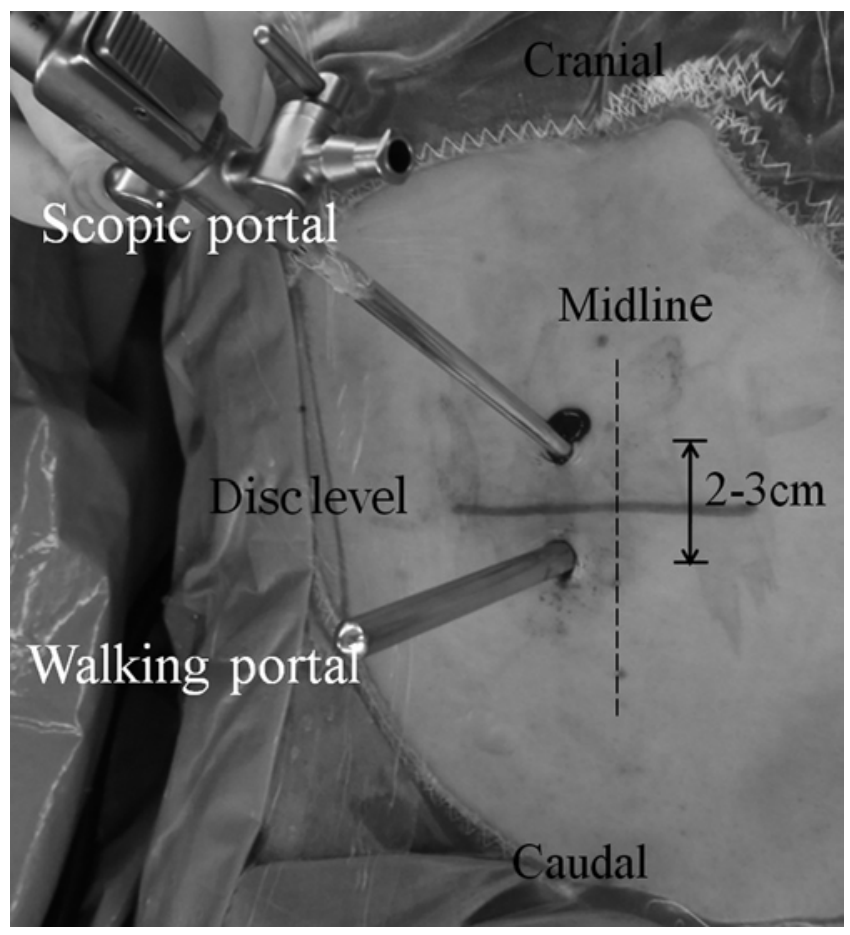

FIG. 2. Portal placements for the unilateral biportal endoscopic approach. The first incision for the working portal (caudal) was vertically placed over the target point. The second incision for the endoscopic portal (cranial) was made 2-3 cm above the upper edge of the first incision. 
connected to the endoscope and set to a pressure of 20-30 $\mathrm{mm} \mathrm{Hg}$ during the procedure; a continuous flow of saline solution irrigation saline is essential to prevent excessive elevation of the epidural pressure. Surgical instruments were inserted through the caudal working portal.

After triangulation with the endoscope and instrument and control of minor bleeding, radiofrequency probes were used for debridement of the soft-tissue remnants overlying the lamina and ligamentum flavum. Following complete exposure of the lower lamina and ligamentum flavum in the targeted interlaminar space, an ipsilateral partial laminotomy was performed under magnified endoscopic vision, using a $3.5-\mathrm{mm}$ drill with soft tissue protection and Kerrison punches (Fig. 3). The endoscopic anatomical view was very similar to the microscopic view of a posterior midline unilateral laminotomy. The ipsilateral ligamentum flavum was removed until full mobilization of the lateral border of the nerve root was achieved. The upper border of the lower lamina is removed for the ipsilateral foraminotomy as needed. Contralateral decompression can be performed at high magnification and with a good endoscopic field of vision (Figs. 4 and 5). Complete removal of the contralateral ligamentum flavum and sublaminar decompression were performed using a Kerrison punch and curette. The endoscope was moved to the contralateral side by taking advantage of muscle and skin elasticity rather than adjusting the patient's position or performing additional skin incisions. Contralateral decompression was performed until the contralateral descending nerve root was identified and decompressed (Fig. 5). If a patient is symptomatic and has ipsilateral disc herniation, it is possible for the surgeon to perform a discectomy under endoscopic view. The level of neural decompression was assessed by normal respiratory-induced dural pulsation and confirmed with endoscopic viewing and use of a blunt probe (Fig. 5). Epidural bleeding was controlled by

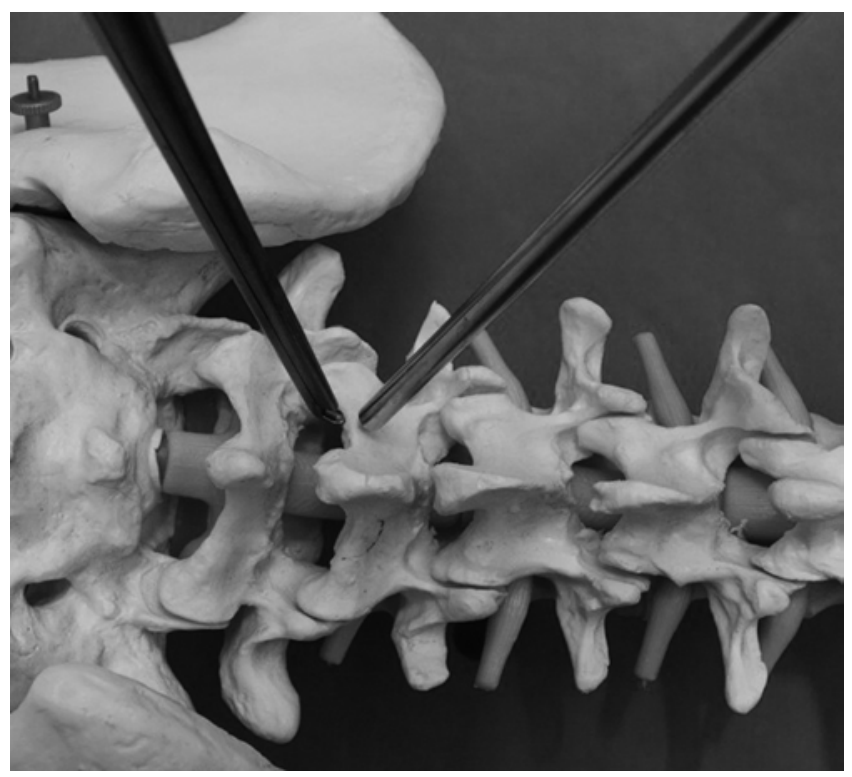

FIG. 3. Triangulation with the endoscope and instrument. Laminotomy was performed under endoscopic view.

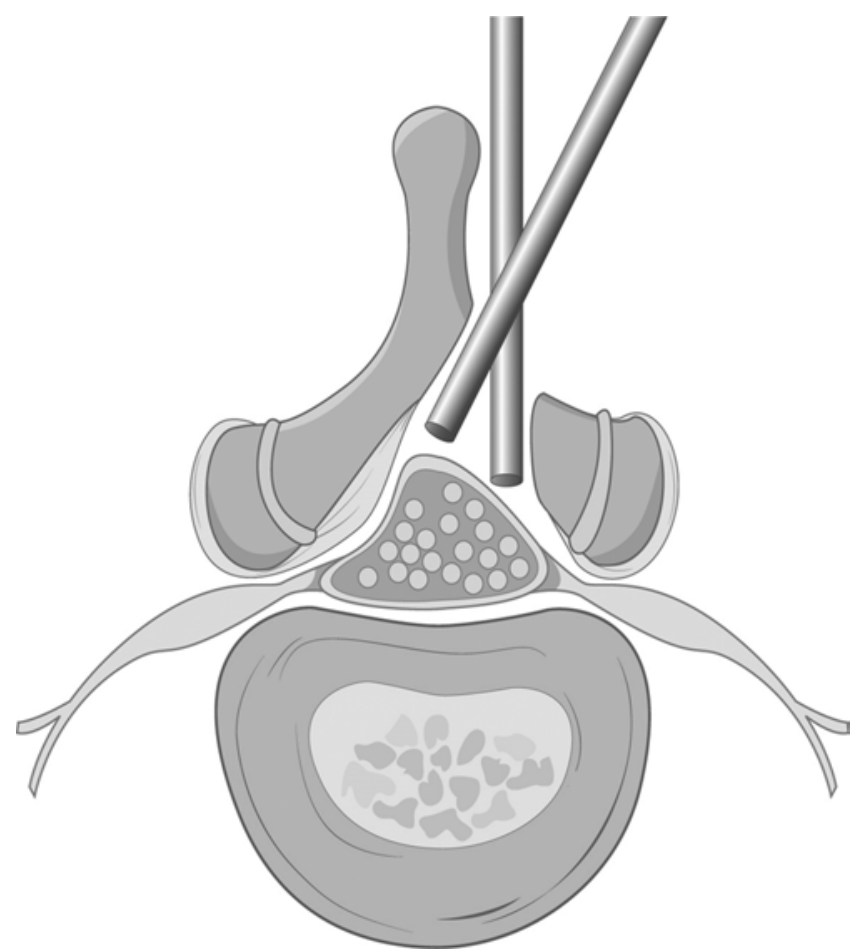

FIG. 4. The endoscopic system allows good visualization of the contralateral foraminal area. Copyright Dong Hwa Heo. Published with permission.

adjusting the pump pressure and coagulation with flexible radiofrequency probes. The skin incisions were closed after removal of the instruments and endoscope (Video 1).

VIDEO 1. The video presentation of percutaneous biportal endoscopic decompression for lumbar stenosis. Ipsilateral partial laminotomy was performed under magnified endoscopic vision, with a $3.5-\mathrm{mm}$ soft tissue protected drill and Kerrison punches. The ipsilateral ligamentum flavum was removed until full mobilization of the lateral border of the nerve root was achieved. In addition, complete removal of the contralateral ligamentum flavumand sublaminar decompression were carried out with a Kerrison punch and curette under endoscopic vision. Copyright Leon Wiltse Memorial Hospital. Published with permission. Click here to view.

\section{Patient Population and Clinical Outcome Analysis}

Our inclusion criteria were as follows: single-level lumbar spinal stenosis, neurological intermittent claudication or radicular leg pain refractory to conservative management for at least 12 weeks, and the absence of significant instability such as spondylolisthesis. We excluded patients with instability, multilevel spinal stenosis, previous lumbar surgery, spinal infections, ossification of the ligamentum flavum, and traumatic lesions such as compression fractures. We enrolled only patients who underwent follow-up for more than 12 months after PBED. A total of 96 patients have prospectively undergone PBED since March 2012. Among them, 58 were enrolled in our study. We performed a retrospective review of our case series. The following clinical parameters were assessed: Oswestry Disability Index (ODI), visual analog scale (VAS) score for leg pain, modified Macnab criteria (excellent, good, 


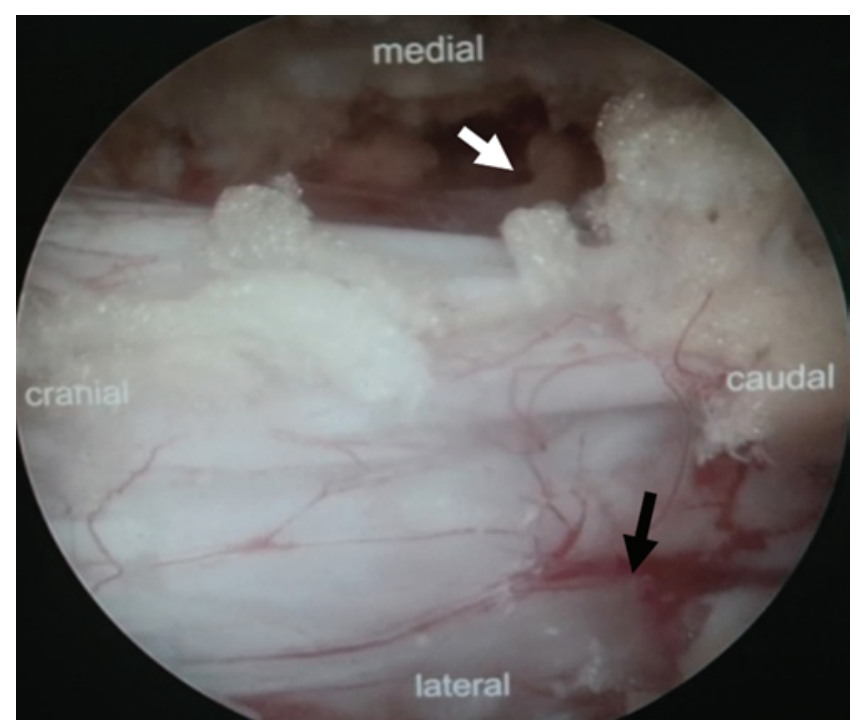

FIG. 5. Intraoperative endoscopic image. Full decompression of the central and bilateral traversing roots was achieved (black arrow, ipsilateral root, white arrow, contralateral root). See Video. Figure is available in color online only.

fair, and poor), operative time, and complications related to the operation. Preoperative and postoperative ODI and VAS at final follow-up were compared. We performed this investigation in accordance with our institutional guidelines, which comply with international laws and policies (institutional review board of The Leon Wiltse Memorial Hospital).

\section{Statistical Analysis}

Statistical analysis was performed using the Wilcoxon rank sum test; $\mathrm{p}<0.05$ was considered statistically significant. We used R 3.1.2 for Windows for the statistical analysis.

\section{Results}

We performed bilateral decompression via a unilateral approach using percutaneous biportal endoscopy (Figs. 5 and 6). The mean operative time for 1 level was $68.9 \pm$ 16.1 minutes. The study participants comprised 18 men and 40 women. The mean age was $63.4 \pm 7.4$ years. The mean follow-up period was $13.8 \pm 3.3$ months. The disc levels that were operated on ranged from L3-4 to L5-S1: L3-4 in 9 cases; L4-5 in 44 cases; and L5-S1 in 5 cases (Table 1). The postoperative mean ODI was significantly improved from $67.2 \pm 11.7$ to $24.3 \pm 8.5$ at final follow-up $(\mathrm{p}<0.05)$ (Table 2). There was a significant decrease in postoperative mean VAS for leg pain from $8.3 \pm 1.1$ to 2.4 \pm 1.1 at final follow-up $(\mathrm{p}<0.05)$ (Table 2). Based on the Macnab criteria, 47 of the 58 patients $(81.0 \%)$ had a good or excellent result, 7 patients had a fair result (12.1\%), and 4 patients had a poor result (6.9\%). One of the patients who had poor results was found to have diabetic neuropathy following a postoperative electrophysiological study, and another patient had degenerative scoliosis. The other 2 patients had incomplete decompression of the contralateral area, which was revealed by postoperative MRI. One
TABLE 1. Patient characteristics*

\begin{tabular}{lc}
\hline \multicolumn{1}{c}{ Characteristics } & Value \\
\hline Mean age (yrs) & $63.4 \pm 7.4$ \\
\hline Sex (M/F) & $18 / 40$ \\
\hline Mean follow-up period (mos) & $13.8 \pm 3.3$ \\
\hline Disc level treated & 9 \\
\hline L3-4 & 44 \\
\hline L4-5 & 5 \\
\hline L5-S1 & 20 \\
\hline Macnab criteria & 27 \\
\hline Excellent & 7 \\
\hline Good & 4 \\
\hline Fair & $68.9 \pm 16.1$ \\
\hline Poor & 3 \\
\hline Mean operative time (mins) & 2 \\
\hline Postop complications & 2 \\
\hline Postop headache & 1 \\
\hline Dural tear & \\
\hline Transient leg numbness & \\
\hline Postop hematoma &
\end{tabular}

* Values are number of patients unless stated otherwise. Mean values are presented as \pm SD.

patient received a transforaminal lumbar interbody fusion due to incomplete decompression and foraminal stenosis 13 months postoperatively.

We converted from the PBED procedure to conventional microscopic surgery in 3 patients during the learning curve of 20 cases. Endoscopic surgery was converted to open microscopic surgery in 2 patients with severe dura adhesions of the ligament flavum, and 1 patient with dural tearing during our early experience.

There were no serious complications related to surgery. Accidental dural tears occurred in 2 patients. One patient was treated with primary sutures under microscopy, and the other patient was treated by clipping under endoscopy. Three patients complained of postoperative headache and neck pain, which was managed with analgesics and bed rest. Postoperative headache occurred after using a large volume of irrigation fluid in 2 patients and after a dura tear and CSF leakage in 1 patient. Transient numbness of the leg occurred in 2 patients, and postoperative epidural hematoma developed in 1 patient; these 2 conditions spontaneously resolved with conservative management.

\section{Discussion}

Although a conventional open decompressive laminectomy is a standard and effective treatment for lumbar stenosis, anatomical supporting tissues, such as muscles and

TABLE 2. Preoperative and postoperative clinical parameters

\begin{tabular}{ccc}
\hline \multicolumn{1}{c}{ Scale* $^{*}$} & Preop & Postop ( $\geq 6$ mos) \\
\hline ODI & $67.2 \pm 11.7$ & $24.3 \pm 8.5$ \\
\hline VAS for leg pain & $8.3 \pm 1.1$ & $2.4 \pm 1.1$ \\
\hline${ }^{*} p<0.05$. & &
\end{tabular}




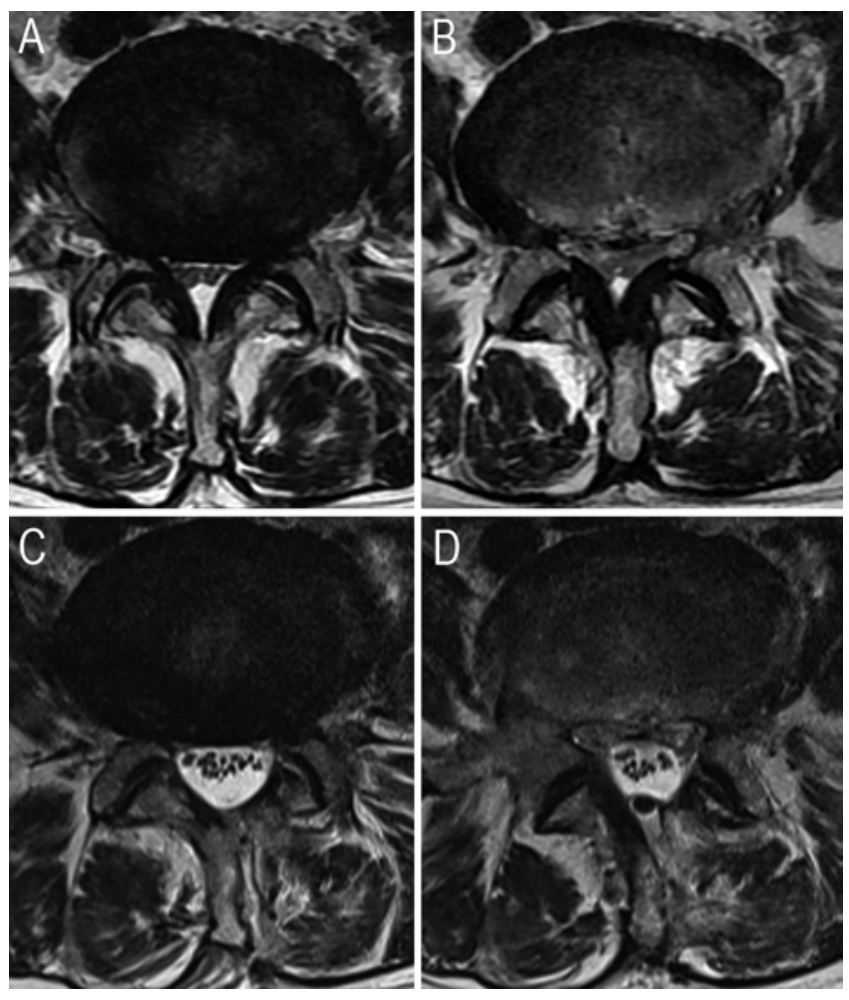

FIG. 6. Preoperative MR images showing lumbar stenosis at L4-5 (A and $B$ ). Postoperative MR images showing that the spinal canal was well decompressed (C and D).

ligaments, can be negatively affected by open surgery. ${ }^{9}$ Surgical damage of surrounding tissues can lead to postoperative back pain and muscle atrophy. ${ }^{4,9}$ Moreover, secondary postoperative instability after decompression can cause pain, and additional fusion surgery may be needed. ${ }^{2}$ As a result, minimally invasive surgical techniques have been developed to reduce the damage to surrounding tissues. ${ }^{2,9-11}$ Microscopic unilateral laminotomy with bilateral decompression via a unilateral approach has been used in degenerative lumbar stenosis, with good postoperative outcomes reported. However, disadvantages of a microscopic approach include the need for dissection of muscle and minor difficulties in contralateral visualization in patients who are obese or stocky. Additionally, as the microscopic approach restricts the surgeon's view to outside the spinal canal and limits the range of motion for surgical instruments, an extensive laminectomy and adjustment of the patient's position may be necessary to achieve the necessary decompression of the contralateral traversing nerve root during the procedure. Recently, single-portal endoscopic decompression has been attempted. ${ }^{12}$ However, surgeons have yet to become familiar with the single-portal endoscopic decompression approach, and incomplete decompression is a possible outcome.

The PBED approach combines the advantages of standard open surgery and endoscopic spinal surgery. This technique is a modification and fusion of translaminar endoscopic decompression and microscopic unilateral laminotomy with bilateral arthroscopic decompression. The PBED approach allows the surgical area to be viewed at high magnification and enables a good field of vision of the contralateral, sublaminar, and foraminal areas (Figs. 4 and 5). The contralateral sublaminar space can be easily viewed by shifting the endoscope without the need for additional skin incisions or an adjustment of the patient's position (Figs. 4 and 5). Moreover, the surgical endoscopic view is similar to that in conventional microscopic surgery. Therefore, the anatomical view of PBED will be familiar to the spinal surgeon, which may help in learning the technique. Percutaneous biportal endoscopy is similar to a microendoscopic tubular decompression approach, with the advantages of easier bilateral foraminal decompression as a result of unrestricted use of the tubular retractor system and less bleeding due to continuouspressure saline irrigation.

A higher risk of dural tears is one of the disadvantages of microscopic and microendoscopic tubular decompression decompressive surgery. ${ }^{5,10}$ In comparison, PBED allows the surgical field to be viewed at high magnification, and the fluid from continuous pressure irrigation enables slight compression of the dura mater and widening of the contralateral epidural space during procedures (Figs. 4 and 5). Therefore, we suggest that contralateral decompression may be easier to perform and has a lower risk of dural tears.

Interlaminar endoscopic decompression surgery can also result in complications such as dural tears, epidural hematoma, neural injury, and inferior facet fracture. ${ }^{12} \mathrm{We}$ believe that it may also be more difficult to manipulate surgical instruments, such as the punch and high-speed drill, compared with microscopic surgery, and that there is a longer learning curve for the 1-portal endoscopic system. The concept of a 2-portal endoscopic system is different than single-portal endoscopic interlaminar decompression surgery. Two skin portals are made: one portal is used for the endoscope and the other permits entry of the surgical instruments. Therefore, the endoscopic system is similar to joint arthroscopy and uses a triangulation approach (Figs. $1 \mathrm{~A}$ and 3 ). The 2 portals are ipsilateral and the endoscope meets with surgical instruments at the interlaminar area. Therefore, handling and movement of instruments is unrestricted, similar to microscopic surgery. Standard laminectomy instruments can be inserted through the working portal. Therefore, the organizational format of the endoscopic operating procedure is similar to open microscopic surgery, but without retraction of muscle. Unilateral biportal endoscopy enables a good field of vision of ipsilateral and contralateral spinal canal anatomy.

During the PBED learning curve, operation time was long, and the volume of irrigation saline was much greater compared with those of more recent operations. Although continuous saline irrigation enables good visualization and reduces intraoperative bleeding, excess irrigation may induce meningeal irritation and consequent headache. ${ }^{1,7}$ In this study, 2 patients complained of postoperative headache and neck pain without CSF leakage. Fortunately, postoperative headaches did not occur in patients who were operated on more recently in a shorter period of time.

In this study, we were only able to assess and follow up 66 patients. Therefore, the results cannot be extrapolated. To improve assessment of the clinical outcomes of PBED, a larger number of patients should be studied and followed up. 


\section{Conclusions}

The anatomical view of PBED is very similar to that of conventional open surgery and allows for good visualization of the contralateral, sublaminar, and medial foraminal areas. We suggest that PBED may be an alternative and minimally invasive procedure for treatment of degenerative lumbar stenosis.

\section{References}

1. Choi G, Kang HY, Modi HN, Prada N, Nicolau RJ, Joh JY, et al: Risk of developing seizure after percutaneous endoscopic lumbar discectomy. J Spinal Disord Tech 24:83-92, 2011

2. Costa F, Sassi M, Cardia A, Ortolina A, De Santis A, Luccarell G, et al: Degenerative lumbar spinal stenosis: analysis of results in a series of 374 patients treated with unilateral laminotomy for bilateral microdecompression. J Neurosurg Spine 7:579-586, 2007

3. De Antoni DJ, Claro ML, Poehling GG, Hughes SS: Translaminar lumbar epidural endoscopy: anatomy, technique, and indications. Arthroscopy 12:330-334, 1996

4. Hu ZJ, Fang XQ, Zhou ZJ, Wang JY, Zhao FD, Fan SW: Effect and possible mechanism of muscle-splitting approach on multifidus muscle injury and atrophy after posterior lumbar spine surgery. J Bone Joint Surg Am 95:e192, 2013

5. Ikuta K, Tono O, Tanaka T, Arima J, Nakano S, Sasaki K, et al: Surgical complications of microendoscopic procedures for lumbar spinal stenosis. Minim Invasive Neurosurg 50:145149, 2007

6. Javid MJ, Hadar EJ: Long-term follow-up review of patients who underwent laminectomy for lumbar stenosis: a prospective study. J Neurosurg 89:1-7, 1998

7. Joh JY, Choi G, Kong BJ, Park HS, Lee SH, Chang SH: Comparative study of neck pain in relation to increase of cervical epidural pressure during percutaneous endoscopic lumbar discectomy. Spine (Phila Pa 1976) 34:2033-2038, 2009

8. Martin BI, Mirza SK, Comstock BA, Gray DT, Kreuter W, Deyo RA: Reoperation rates following lumbar spine surgery and the influence of spinal fusion procedures. Spine (Phila Pa 1976) 32:382-387, 2007

9. Mobbs RJ, Li J, Sivabalan P, Raley D, Rao PJ: Outcomes after decompressive laminectomy for lumbar spinal stenosis: comparison between minimally invasive unilateral laminectomy for bilateral decompression and open laminectomy: clinical article. J Neurosurg Spine 21:179-186, 2014

10. Podichetty VK, Spears J, Isaacs RE, Booher J, Biscup RS: Complications associated with minimally invasive decompression for lumbar spinal stenosis. J Spinal Disord Tech 19:161-166, 2006

11. Poletti CE: Central lumbar stenosis caused by ligamentum flavum: unilateral laminotomy for bilateral ligamentectomy: preliminary report of two cases. Neurosurgery 37:343-347, 1995

12. Sairyo K, Sakai T, Higashino K, Inoue M, Yasui N, Dezawa A: Complications of endoscopic lumbar decompression surgery. Minim Invasive Neurosurg 53:175-178, 2010

\section{Disclosures}

The authors report no conflict of interest concerning the materials or methods used in this study or the findings specified in this paper.

\section{Author Contributions}

Conception and design: Heo, Eum, Son. Acquisition of data: Heo, Eum. Analysis and interpretation of data: all authors. Drafting the article: Heo, Eum. Critically revising the article: Heo, Eum, Park. Reviewed submitted version of manuscript: Heo, Eum, Park. Approved the final version of the manuscript on behalf of all authors: Heo. Statistical analysis: Heo, Eum. Administrative/ technical/material support: Heo, Eum, Son. Study supervision: Heo, Park.

\section{Supplemental Information \\ Videos}

Video 1. https://vimeo.com/142142258.

\section{Correspondence}

Dong Hwa Heo, Department of Neurosurgery, The Leon Wiltse Memorial Hospital, 994-3, Ingye-dong, Paldal-gu, Suwon-si, Gyeonggi-do 442-833, Korea. email: youmans@empal.com. 